\title{
ARTÍCULOS
}

\section{FACTORES CLAVE PARA EL FUTURO DE LA ARCHIVÍSTICA}

\author{
María-Paz Martín-Pozuelo, Manuela Moro-Cabero y Cristina Sanz-Baena
}
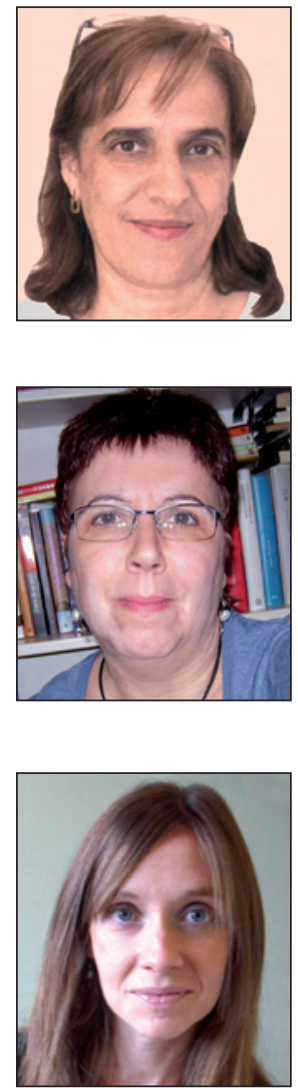

María-Paz Martín-Pozuelo, doctora por la Univ. de Alcalá, es profesora titular en el Departamento de Biblioteconomía de la Univ. Carlos III de Madrid (UC3M). Ha sido ponente invitada en congresos nacionales e internacionales. Fundó el Laboratorio de investigación archivística de la UC3M dentro del cual dirige el Observatorio de Prospectiva Archivística y Sociedad, en el que participan universidades españolas y extranjeras. Es autora de publicaciones sobre la construcción teórica de la archivística, su impacto en la sociedad y su futuro científico y profesional.

Universidad Carlos III de Madrid paz.martin-pozuelo@uc3m.es http://observatoriodeprospectivaarchivistica.blogspot.com

Manuela Moro-Cabero, doctora por la Univ. de Salamanca, es profesora titular de gestión de documentos de archivo en el Depto. de Biblioteconomía y Documentación de dicha universidad. Es miembro del Observatorio de Prospectiva Archivística y Sociedad de la Univ. Carlos III de Madrid y del Comité Técnico CT50 de la Asociación Española de Normalización y Certificación (Aenor), donde coordina el Grupo de Trabajo de Evaluación del Subcomité de Gestión de Documentos y Aplicaciones (SC1). Ha publicado sobre gestión de documentos de archivo, gestión normalizada de documentos, calidad y archivos.

Universidad de Salamanca moroca@usal.es

Cristina Sanz-Baena es licenciada en periodismo por la Universidad Complutense de Madrid y experta universitaria en protocolo y ceremonial por la Universidad Miguel Hernández de Elche. Participa en el Programa de Doctorado en Documentación: Archivos y Bibliotecas en el Entorno Digital, de la Universidad Carlos III de Madrid. Es miembro del Observatorio de Prospectiva Archivística y Sociedad en el que coordina la mesa de trabajo Archivística y Tecnología. Desarrolla su trabajo en la Unidad de Gestión de Recursos de la Información del Centro de Investigaciones Energéticas, Medioambientales y Tecnológicas (Ciemat).

Centro de Investigaciones Energéticas, Medioambientales y Tecnológicas (Ciemat) cristina.sanz@ciemat.es

\section{Resumen}

Se identifican y priorizan factores clave para el futuro de la archivística a partir de las opiniones de un grupo reducido de profesionales e investigadores implicados en la archivística, mediante la técnica cualitativa de búsqueda de consenso. El estudio ha permitido identificar 70 factores distribuidos entre las cinco variables analizadas: futuro del archivo (26), de la archivística (10), del profesional (12), de las relaciones con la tecnología (10) y del futuro social (12). La cantidad, el alcance y los resultados obtenidos invitan a dar continuidad a la investigación en otros contextos más amplios así como con mayores grupos de expertos provenientes de diferentes sectores.

\section{Palabras clave}

Archivística, Gestión de documentos, Método prospectivo, Estudios de futuro, Investigación cualitativa, Técnicas de consenso.

\section{Title: Key factors for the future of Archival science}

\begin{abstract}
Key factors for the future of Archival science are identified and prioritized from the views of a small group of professionals and researchers, using the qualitative technique of consensus-building. The study has identified 70 factors distributed among five variables: the future of archives (26), of Archival science (10), of professionals (12), of relationships with technologies (10) and of society (12). The amount, scope and results invite us to continue the research in broader contexts as well as in larger groups of experts from different sectors.
\end{abstract}




\section{Keywords}

Archival science, Document management, Prospective methods, Future studies, Qualitative research, Consensus techniques.

Martín-Pozuelo, María-Paz; Moro-Cabero, Manuela; Sanz-Baena, Cristina. "Factores clave para el futuro de la archivística". El profesional de la información, 2011, marzo-abril, v. 20, n. 2, pp. 135-141.

DOI: 10.3145/epi.2011.mar.02

\section{Introducción}

Desde que se inicia el nuevo milenio, diferentes investigaciones han ido revelando la necesidad de actuar de inmediato sobre diferentes aspectos de la archivística que demandan, cuando menos, una revisión de sus presupuestos y de su metodología. Entre las preocupaciones viene ocupando un lugar destacado el impacto que sobre la archivística tienen las tecnologías (Gilliland-Swetland, 2000; Cook, 2001; Duranti, 2001; Martín-Pozuelo, 2001; Borglund, 2005). Son significativos los estudios que revelan la necesidad de un cambio de paradigma (Cook, 2001; Ribeiro, 2001). De índole más global y filosófica, destacamos aquí las reflexiones que diferentes autores vierten en un número de la revista Tabula (2007) dedicado a la archivística de la post-modernidad (Brothman, 2007; Cook, 2007; Harris, 2007; Keteelaar, 2007; Schwart, 2007; Greene, 2007; Nesmith, 2007; Jimerson, 2007).

Sin embargo faltan investigaciones que aborden el futuro desde un análisis riguroso con técnicas apropiadas. Desde una mirada prospectiva, y siguiendo para ello la metodología del mismo nombre (Martín-Pozuelo, 2010), el Observatorio de Prospectiva Archivística y Sociedad (en adelante OPAS) ha planteado el I Estudio prospectivo sobre el futuro de la archivística (en delante I Estudio...) que se propone concluir con el diseño de los escenarios de futuro para la archivística. Está planteado en tres fases, a saber: identificación de factores clave, identificación de tendencias y diseño de escenarios de futuro.

En este artículo se presentan la metodología y los resultados del ensayo para la ejecución de la primera fase; esto es, la identificación de los factores clave. La investigación ha sido realizada con un grupo reducido de expertos y, en atención a los resultados obtenidos, más adelante se aplicará en un marco geográfico más amplio.

Se pretende responder a la siguiente pregunta: ¿Qué factores críticos determinarán en un futuro a corto y medio plazo el desarrollo de la archivística?

\section{El futuro de la archivística: un dilema complejo}

La investigación sobre el futuro es siempre un difícil examen de incertidumbres. Un correcto enfoque nos permitirá obtener evidencias relevantes que, en opinión de algunos teóri- cos, favorecerán una actuación sobre el tema tratado (Medina-Vásquez; Ortegón, 2006; Waardenburg, 1999, p. 663). En la archivística la dificultad en el análisis responde a varias razones:

- el marco teórico: ninguna teoría nos permite encuadrar el análisis;

- el objeto: no es un objeto único ni invariable. Se trata de un objeto múltiple: futuro de la ciencia, del profesional, de sus relaciones, de su entorno y de todos los factores externos que inciden sobre ella. A su vez, todos estos factores tienen una presencia y un comportamiento desigual por espacios geográficos y tipos de administración;

- el método: en archivística por el momento no se realizan experiencias de aplicación de métodos prospectivos.

Diferentes investigadores aconsejan revisar de inmediato los presupuestos y métodos de la archivística

\section{Justificación}

A pesar de estas dificultades, el modo en que los cambios -en particular los tecnológicos y sociales- están impactando sobre la ciencia archivística justifica sobradamente esta investigación que, aún a riesgo de ser imperfecta, resulta imprescindible por tres razones de consideración:

a) colaborar en la creación de una cultura prospectiva, ensayando para ello el método prospectivo en el contexto de la archivística;

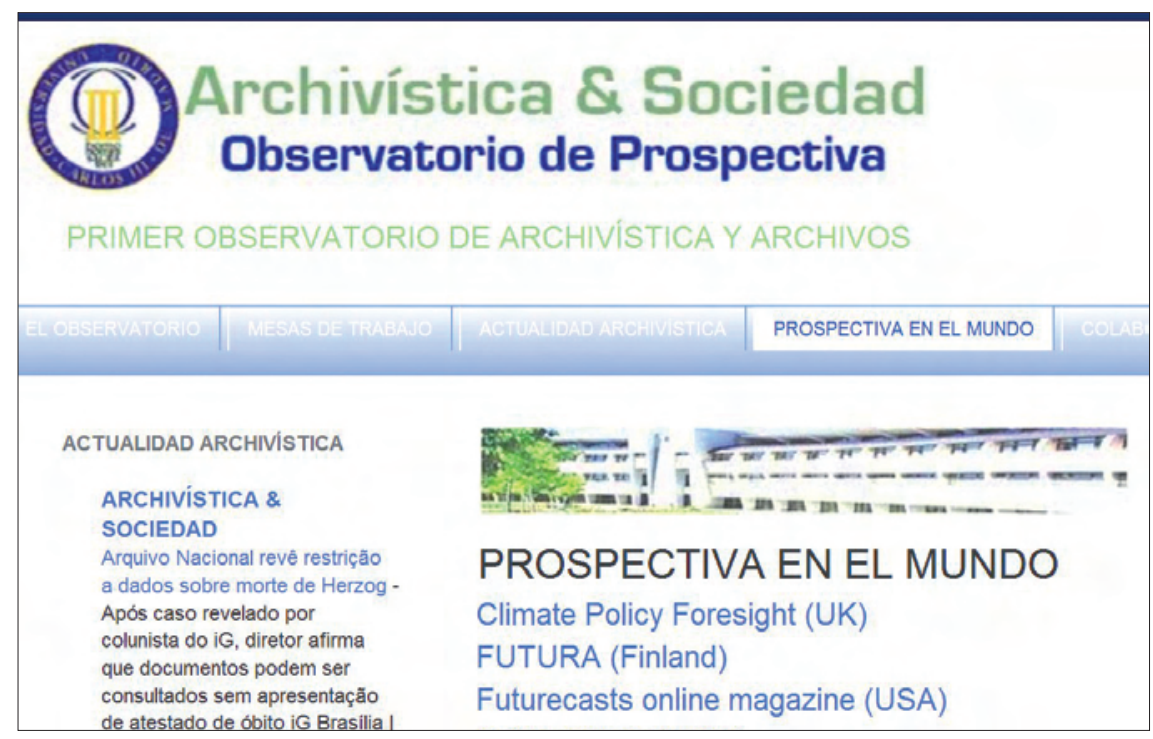

http://observatoriodeprospectivaarchivistica.blogspot.com/ 
b)disponer de una primera lista de factores que permita identificar las tendencias en la disciplina y en sus profesionales, sus relaciones con el entorno, etc.

c) poder proceder en una fase final a la construcción de los escenarios de futuro.

\section{Contexto de la investigación: el OPAS}

La investigación ha sido planteada y desarrollada en el marco del citado OPAS, nacido para estudiar las relaciones de los archivos, la archivística y la gestión de documentos con la sociedad. Pretende, igualmente, reunir evidencias a partir de las cuales poder estudiar el futuro de la ciencia archivística, su objeto de estudio y el sujeto que la hace posible. A tal fin el Observatorio tiene abiertos cuatro diferentes capítulos de observación y análisis: área científica, área tecnológica, área social y área profesional. Con carácter claramente exploratorio este artículo se plantea para iniciar cada uno de estos cuatro capítulos que más tarde irán siendo analizados con proyectos específicos y forma parte del I Estudio prospectivo.

\section{Finalidad y objetivos}

Además de identificar los factores críticos y su orden de prioridad, el estudio también ha ensayado el proceso y las técnicas prospectivas que han posibilitado la obtención y el consenso de respuestas, creando con ello cultura prospectiva en la comunidad archivística.

Los cuatro elementos clave sobre los que se construye toda ciencia son:

- la propia ciencia (archivística),

- su objeto (el archivo),

- el sujeto que la hace posible (el archivero) y

- el entorno en el que se inscribe y del que recibe constantes influencias (como la tecnología y la sociedad).

\section{Material y métodos}

Se ha aplicado el método para el diseño y la construcción de escenarios de futuro que es, quizá, uno de los más y mejor estudiados (Schwartz, 1993; Van der Heijden, 1996; Godet y Roubelat, 1996; Ringland, 1998; Godet, 2004). Siguiendo a este último (Godet, 2000, p. 17) se entiende por escenario el conjunto formado por la descripción de una situación futura y un camino de acontecimientos que posibilitan pasar de una situación original a otra futura. Los escenarios son múltiples.

¿Qué factores críticos determinarán en el futuro el desarrollo de la ciencia archivística, de los archivos y de sus profesionales?

\section{Descripción de la investigación}

\section{a) Elaboración de material preliminar}

Dada la inexperiencia en la aplicación de esta técnica, a los expertos se les proporcionó un dossier que contenía un ejemplar del Proyecto del I Estudio... con detalle de objeti- vos y metodología; algunos escritos prospectivos en otras áreas de conocimiento, con el fin de ilustrar el proceso; y una breve guía metodológica.

\section{b) Selección y primeros contactos con los expertos}

Se consideró esencial realizar la investigación con expertos procedentes de dos sectores: la universidad y la profesión. En la medida de lo posible, se seleccionaron los que reiteradamente hubieran manifestado preocupación e interés por los nuevos retos de la archivística. Se procuró que hubiera representación de archivos administrativos (4) e históricos (2), así como de otros países (Brasil y México, además de España). Se demandó la colaboración de expertos próximos al Observatorio, garantizando así una comprensión más o menos inmediata del ensayo.

El grupo quedó finalmente constituido por diez personas que en su totalidad reunían los condicionantes que se exponen a continuación:

- responsabilidades de administración y dirección en el momento de la investigación;

- amplia experiencia de participación en eventos de carácter científico;

- publicaciones de impacto en el área; e

- integración en algún grupo de trabajo (requisito que cumplían todos salvo uno).

Tras su selección y los pertinentes contactos, telefónico (breve información) y mail (exposición de objetivos de investigación con detalle), les fueron remitidos:

- una invitación formal

- un documento/contrato que debían devolver firmado con el compromiso de permanecer en la investigación hasta el final, salvo imponderables.

- un borrador del proyecto con detalle de objetivos y planificación.

El grupo final quedó reducido a 8 miembros.

\section{c) Segundo contacto con los expertos}

Cerrado el acuerdo de investigación se les remitieron por email cinco plantillas con las preguntas:

¿Qué factores críticos considera usted que determinarán en un futuro a corto y medio plazo:

- la ciencia archivística? (FC)

- el desarrollo del archivo? (FA)

- el desarrollo del profesional de archivos? (FP)

- las relaciones del archivo con la sociedad? (FS)

- el futuro tecnológico del archivo? (FT)

Las cinco plantillas contenían una primera y mínima relación de factores como ejemplo.

\section{d) Primera ronda de resultados}

El objetivo de esta primera ronda era elaborar un listado exhaustivo de factores sobre los que trabajar en sucesivas series. Aunque en la programación inicial se estableció un plazo de 30 días para la realización de esta primera fase, las dificultades de tiempo que muchos de los expertos manifestaron en el primer contacto telefónico, obligaron a ampliar el periodo otros 60 días. Finalmente la información 
fue recolectada entre los días 1 de febrero y 30 de abril de 2009.

\section{e) Segunda ronda de resultados}

Una vez que los ocho participantes elaboraron sus cinco primeros listados de factores críticos se elaboró un listado único para cada una de las cuestiones planteadas.

El producto resultante fue remitido a los expertos para que cada una de ellos considerase la posibilidad de variar su primer listado, a la luz de las aportaciones del resto. Las enumeraciones recibidas en esta segunda recolecta reflejaron idénticos resultados.

\section{f) Tercera ronda de resultados}

Los listados fueron remitidos nuevamente en formato de tabla, a la que se añadió una tercera columna donde los expertos debían priorizar cada uno de los factores. Asignaron valores en orden ascendente de mayor importancia a menor (el valor 1 es considerado más relevante, el 2 menos, etc.). Los factores que se juzgaran sin ninguna o mínima relevancia se dejaban en blanco. El orden de prioridad concedido por cada uno de estos ocho expertos fue muy similar.

Con el fin de acabar de asegurar el consenso sobre algunas cuestiones mínimas observadas, fue elaborado un nuevo documento con los factores ordenados por prioridad, que fue remitido nuevamente para su consideración final. En él fue añadido un apartado para la inclusión de observaciones.

\section{g) Elaboración de resultados finales}

Las tablas que figuran en el apartado de resultados finales muestran la variedad de factores identificados, con su orden de prioridad. Se discuten en el epígrafe siguiente.

\section{Resultados finales}

\section{Identificación de factores clave}

En la tabla 1 se muestra el total de factores identificados por cada uno de los expertos (Exp1/Exp 8) para cada una de las variables definidas (FC; FA, etc.).

Fueron identificados 70 factores correspondientes a las variables: Futuro del archivo (26), de la ciencia archivística (10), del profesional (12), de las relaciones con la tecnología (10) y del futuro social (12).

Algunos de los factores recogidos en la variable FA (futuro del archivo) se repiten en otras variables (futuro tecnológico, futuro profesional...), pero son considerados indepen- dientes en tanto que a juicio de los expertos su incidencia sobre las variables también lo es. Por ejemplo el factor Visibilidad se entiende de modo diferente si se asocia al archivo o al profesional; o el factor Presupuestos difiere si se trata en investigación, en el archivo o en la carrera profesional.

\section{Priorización de factores}

El proceso de priorización se centró especialmente en el debate que suscitaron las primeras propuestas individuales hasta obtener finalmente un listado consensuado. Aquellos factores votados por menos de un $50 \%$ del total fueron descartados.

La técnica prospectiva basada en el consenso permite identificar y priorizar factores clave para la anticipación del futuro

\section{Futuro de la archivística}

En la tabla 2 se presentan los factores clave para el futuro de la archivística, ordenados según la prioridad adjudicada por los expertos.

En la tabla 3 los expertos resaltan tres factores relevantes de incidencia en el futuro de la ciencia archivística: formación (FC-01, FC-09 y FC-10), investigación (FC-02, FC-03, FC-04, FC-09, FC-10) y colaboración (FC-04, FC-05, FC-07, FC-08). Un cuarto factor les mereció también la atención: innovación (FC-06). En nuestra opinión éste se subyuga a

\begin{tabular}{|l|l|}
\hline Código & \multicolumn{1}{|c|}{ Factores críticos en orden de prioridad } \\
\hline FC-01 & Formación especializada \\
\hline FC-02 & Apoyo a I+D \\
\hline FC-03 & Apoyo a I+T \\
\hline FC-04 & Creación de grupos de investigación internacionales \\
\hline FC-05 & Colaboración con y de otras áreas de conocimiento \\
\hline FC-06 & Capacidad de adelantarse al futuro \\
\hline FC-07 & Independencia respecto a la documentación \\
\hline FC-08 & $\begin{array}{l}\text { Mayor presencia y colaboración con las disciplinas } \\
\text { documentales }\end{array}$ \\
\hline FC-09 & Formación en investigación \\
\hline FC-10 & Post grados en investigación archivística \\
\hline
\end{tabular}

Tabla 2. Factores críticos con incidencia en el futuro científico (FC) de la archivística

\begin{tabular}{|c|l|c|c|c|c|c|c|c|c|c|}
\hline Código & Variables & Exp1 & Exp2 & Exp3 & Exp4 & Exp5 & Exp6 & Exp7 & Exp8 & Total \\
\hline FC & Futuro científico de la archivística & 7 & 8 & 7 & 9 & 9 & 6 & 7 & 10 & 10 \\
\hline FA & Futuro del archivo & 21 & 20 & 22 & 21 & 24 & 26 & 21 & 25 & 26 \\
\hline FP & Futuro del profesional & 10 & 11 & 12 & 12 & 11 & 10 & 12 & 9 & 12 \\
\hline FT & Futuro tecnológico & 9 & 9 & 8 & 10 & 11 & 10 & 9 & 10 & 10 \\
\hline FS & Futuro social & 8 & 6 & 9 & 9 & 12 & 12 & 9 & 11 & 12 \\
\hline & Total factores & 55 & 54 & 58 & 61 & 67 & 64 & 58 & 65 & 70 \\
\hline
\end{tabular}

Tabla 1. Total de factores críticos identificados

Exp = experto; FC = Futuro científico; FA = Futuro del archivo; FP = Futuro profesional; FT = Futuro tecnológico; FS = Futuro social. En azul se indica el número máximo de factores obtenidos para cada una de las cinco variables. 
los anteriores, dado que la competencia de crear e innovar puede ser desarrollada mediante formación e investigación; en idéntica línea, la colaboración favorece la innovación. EI número de factores clave es reducido puesto que el resto de los aportados matizan o delimitan los anteriores. Sin embargo, el número se eleva considerablemente para el futuro de los archivos, tal y como se refleja en la tabla 3 , donde se aprecian hasta 26 propuestas.

\section{Futuro de los archivos}

Los factores expuestos son variados y se relacionan con el objeto de trabajo (documento FA-01, FA-05 y memoria FA02), con la finalidad (utilidad FA-03, servicio FA-19, FA-17, FA-16, orientación hacia los usuarios FA-08, FA-10, etc.), los recursos tecnológicos, presupuestarios y humanos disponibles y necesarios (FA-07, FA-09, FA-11, FA-14, FA-18, FA-20, FA-22), la administración de unidades, fondos e instrumentos para hacerla factible (FA-06, FA-12, FA-13, FA-19, FA-25, FA-21, FA-26), incluso con su imagen (FA-04, FA-24, FA-11). Destacan, objeto, finalidad e imagen entre los factores más prioritarios, aunque se incluyen numerosos factores destinados al modelo de administración y sus recursos. Se reitera

\begin{tabular}{|c|c|}
\hline Código & Factores críticos en orden de prioridad \\
\hline FA-01 & Generalización de los nuevos documentos \\
\hline FA-02 & Constitución de la memoria: qué documentos conservar \\
\hline FA-03 & Multiplicidad de la utilidad de los archivos \\
\hline FA-04 & Invisibilidad del archivo respecto de la propia institución \\
\hline FA-05 & Volumen de documentación \\
\hline FA-06 & Desarrollo de herramientas específicas de gestión \\
\hline FA-07 & Adecuados recursos personales y financieros \\
\hline FA-08 & El deseo y necesidad de información de los usuarios \\
\hline FA-09 & Financiación pública y privada \\
\hline FA-10 & Adaptación a las necesidades informativas de la sociedad \\
\hline FA-11 & La profesionalización de sus responsables \\
\hline FA-12 & $\begin{array}{l}\text { Adaptación y capacidad de actualización a nuevas } \\
\text { funciones }\end{array}$ \\
\hline FA-13 & Adaptación de los planes y estrategias de desarrollo \\
\hline FA-14 & Las nuevas tecnologías y la sociedad de la información \\
\hline FA-15 & $\begin{array}{l}\text { Los archivos como sujetos activos susceptibles de crear } \\
\text { cultura }\end{array}$ \\
\hline FA-16 & $\begin{array}{l}\text { Insistir sobre la idea de que los archivos nos descubren } \\
\text { el pasado pero hacen también posible la gestión del } \\
\text { presente }\end{array}$ \\
\hline FA-17 & Definición del entorno de trabajo \\
\hline FA-18 & Presupuesto suficiente \\
\hline FA-19 & Definición de objetivos claros y realistas \\
\hline FA-20 & Formación completa en materia documental \\
\hline FA-21 & Colaboración interdisciplinar \\
\hline FA-22 & $\begin{array}{l}\text { Continuidad y seguridad de permanencia de personal y } \\
\text { de los equipos de trabajo }\end{array}$ \\
\hline FA-23 & Trabajo en equipo coordinado y equilibrado \\
\hline FA-24 & Difusión global en entorno web \\
\hline FA-25 & $\begin{array}{l}\text { Aplicación estandarizada de las nuevas tecnologías en la } \\
\text { gestión y difusión }\end{array}$ \\
\hline FA-26 & $\begin{array}{l}\text { Estandarización de las normas básicas aplicables en } \\
\text { todos los aspectos de la gestión }\end{array}$ \\
\hline
\end{tabular}

Tabla 3. Factores críticos con incidencia en el futuro de los archivos (FA) el factor de colaboración interna (FA-23) y externa (FA-21), así como la formación (FA-20).

\section{Futuro profesional}

En cuanto al futuro de la profesión, los factores que sobresalen están relacionados con: formación del profesional (FP-01, FP-02, FP-03, FP-08, FP-11), reconocimiento (FP-04), actuación (FP-05) y condiciones laborales (FP-10, FP-12). EI contexto de cambio y entorno de trabajo electrónico afloran bajo los factores relacionados con formación. La colaboración, y más concretamente en un entorno interdisciplinar, es otro factor que se reitera en esta variable (FP-09).

\section{Futuro tecnológico}

El número de factores clave que inciden sobre el futuro tecnológico desciende sensiblemente respecto a los aportados para el futuro del profesional, tal y como se muestra en la tabla 5.

Los expertos priorizan la conservación de los documentos, su tratamiento y recuperación (FT-01, FT-02, FT-03), la digitalización (FT-05), y, en segundo término, la administración del cambio tecnológico y sus consecuencias (FT-06, FT-07, FT-08, FT-10). Se incluye en esta variable el factor Calidad total que no se relaciona directamente con la misma, y que a nuestro entender estaría mejor ubicado en la variable Futuro de los archivos, donde se ha podido observar la preocupación por los métodos de administración.

Finalmente, en la tabla 6 se enumeran los factores que influyen en el futuro social de la archivística, los archivos y sus profesionales. Curiosamente, esos factores no han sido citados específicamente para los valores FC (ciencia archivística) y FP (profesión) aunque indirectamente influyan tanto en uno como en otro. A destacar factores relativos a la finalidad del archivo como servicio que garantiza los derechos de la ciudadanía y colabora en el logro de un buen gobierno.

\section{Conclusiones}

La investigación realizada ha permitido obtener 70 sugerentes factores, que pueden determinar el futuro y que desve-

\begin{tabular}{|c|l|}
\hline Código & \multicolumn{1}{|c|}{ Factores clave en orden de prioridad } \\
\hline FP-01 & Formación especializada \\
\hline FP-02 & Actualización regular y reglada de conocimientos \\
\hline FP-03 & $\begin{array}{l}\text { Definición de competencias respecto de otros } \\
\text { profesionales por el mercado de trabajo }\end{array}$ \\
\hline FP-04 & Estatuto profesional \\
\hline FP-05 & Ética profesional \\
\hline FP-06 & La revolución tecnológica y su incidencia profesional \\
\hline FP-07 & $\begin{array}{l}\text { El cambio de paradigma de los documentos electrónicos } \\
\text { y su incidencia profesional }\end{array}$ \\
\hline FP-08 & $\begin{array}{l}\text { La necesidad de adaptación, reciclaje y formación } \\
\text { continua en el entorno profesional }\end{array}$ \\
\hline FP-09 & La necesidad de trabajar en grupos interdisciplinares \\
\hline FP-10 & $\begin{array}{l}\text { Condiciones laborales adecuadas: convenientemente } \\
\text { remuneradas y con solución de continuidad. }\end{array}$ \\
\hline FP-11 & Homogenizar la formación \\
\hline FP-12 & Brecha entre la esfera pública y la privada. \\
\hline
\end{tabular}

Tabla 4. Factores críticos con incidencia en el futuro profesional (FP) 


\begin{tabular}{|c|l|}
\hline Código & \multicolumn{1}{|c|}{ Factores clave en orden de prioridad } \\
\hline FT-01 & Conservación de documentos electrónicos \\
\hline FT-02 & Desarrollo de metadatos \\
\hline FT-03 & Recuperación de la información \\
\hline FT-04 & $\begin{array}{l}\text { Calidad total aplicada a los procesos del archivo, tanto de } \\
\text { cara a la gestión como a la difusión }\end{array}$ \\
\hline FT-05 & Digitalización \\
\hline FT-06 & La renovación tecnológica \\
\hline FT-07 & $\begin{array}{l}\text { Cambio de paradigma que supone la gestión de y con } \\
\text { documentos electrónicos }\end{array}$ \\
\hline FT-08 & $\begin{array}{l}\text { Los nuevos soportes y las nuevas herramientas } \\
\text { tecnológicas para la gestión, conservación y acceso }\end{array}$ \\
\hline FT-09 & La estandarización de normas descriptivas y de difusión \\
\hline FT-10 & La informatización integral \\
\hline
\end{tabular}

Tabla 5. Factores críticos con incidencia en el futuro tecnológico (FT)

lan áreas de trabajo, investigación y reflexión que pasamos a enumerar.

1) Se ha demostrado que el empleo de la técnica prospectiva basada en el consenso es eficaz en la identificación y priorización de un conjunto de factores clave para la anticipación del futuro de las variables observadas: ciencia archivística, archivos, profesional, tecnología y perspectiva social.

La técnica empleada ha favorecido el debate sobre cuestiones reales que han tenido que ser consideradas para fijar los factores, que afectan de modo desigual a los archivos públicos y privados. Igualmente, dicha técnica ha permitido el consenso entre expertos provenientes de diferentes sectores, que operan en diversos contextos y deben asumir problemáticas y retos de futuro heterogéneos. En opinión de los expertos participantes se ha demostrado su utilidad para generar reflexión sobre "su realidad" (la de cada uno, que en su cotidianidad suele pasar desapercibida) y las realidades de los restantes expertos. La coincidencia o divergencia observada en dichas realidades reflejan al experto idénticas situaciones experimentadas o nuevas perspectivas. En ambos casos, la percepción final de los expertos es de confianza y de necesidad de anticipación sobre el futuro de los archivos y de la archivística. Asimismo, perciben la idoneidad de la técnica usada para asegurar el proceso identificador.

2) El número de 70 factores críticos justifica sobradamente la elaboración de esta investigación y es indicativo de la riqueza del método, permitiéndonos plantear una futura muestra de mayor alcance que evite posibles sesgos.

3) En la ciencia archivística destacan la formación, la investigación y la colaboración. La formación se reitera en buena parte de las variables (recursos humanos en archivo, profesional y tecnología). Por otro lado, la investigación queda implícita en las propuestas tecnológicas y sociales como requisito necesario para abordar ciertos factores (gestión del cambio, resolución y aplicación de tecnologías, etc.). De igual modo resurge la colaboración como factor a considerar, en la variable archivo y en la variable profesional, tanto desde la perspectiva interna -colaboración en la ejecución del trabajo-, como externa -con una visión multidisciplinar-.

\begin{tabular}{|c|c|}
\hline Código & Factores clave en orden de prioridad \\
\hline FS-01 & El archivo como servicio a la sociedad \\
\hline FS-02 & $\begin{array}{l}\text { Conservación de documentos electrónicos como prueba } \\
\text { social }\end{array}$ \\
\hline FS-03 & Comunicación con el ciudadano \\
\hline FS-04 & Protección de datos \\
\hline FS-05 & Auge del interés social por asuntos patrimoniales \\
\hline FS-06 & Distribución competencial \\
\hline FS-07 & Derecho acceso a la información. \\
\hline FS-08 & $\begin{array}{l}\text { Transparencia de procesos y de gestión de políticas } \\
\text { públicas. }\end{array}$ \\
\hline FS-09 & Coordinación interadministrativa \\
\hline FS-10 & $\begin{array}{l}\text { Coordinación consensuada para el desarrollo y } \\
\text { aplicación de estándares para la difusión }\end{array}$ \\
\hline FS-11 & $\begin{array}{l}\text { Apoyo institucional y económico al desarrollo de } \\
\text { jornadas y eventos para el desarrollo de los archivos, } \\
\text { tanto públicos como privados }\end{array}$ \\
\hline FS-12 & $\begin{array}{l}\text { Soluciones tecnológicas gratuitas para la gestión y } \\
\text { difusión globalizada del Patrimonio y portal de acceso } \\
\text { común }\end{array}$ \\
\hline
\end{tabular}

Tabla 6. Factores críticos con incidencia en el futuro social (FS)

4) Los factores desvelados que inciden en el archivo se circunscriben en orden de prioridad al objeto de trabajo (documento, entendido como evidencia de acciones presentes y como patrimonio o memoria social) y a la finalidad del archivo (servicio a la institución y a la sociedad). La imagen del archivo ocupa el tercer lugar en la importancia temática. A continuación aflora la gestión del archivo aunque cabe señalar que incluye buen número de factores si aunamos la preocupación por los recursos. Este hecho induce a pensar que el archivero prima el objeto y la finalidad frente a la gestión del archivo, aunque sí se interesa por determinados aspectos que lo definen, señalando su posible incidencia de futuro.

5) La formación y el reconocimiento profesional son los aspectos de mayor relevancia para considerar en el futuro profesional. Imagen del archivo y reconocimiento profesional coinciden en rango en diferentes variables. Las competencias profesionales se demandan como apoyo a este reconocimiento y desde la perspectiva social el factor se reitera. Los factores relacionados con las condiciones laborales son ubicados en últimos puestos y reorientados a reconocer desigualdades provenientes de la realidad productiva y social.

6) En la variable tecnológica, la continuidad del objeto de trabajo y su gestión son priorizados ante otros factores de futuro, destacando el afrontar la gestión del cambio tecnológico. A este tenor, formación e investigación se muestran implícitos, obteniendo nuevamente una reiteración en los intereses desvelados por los expertos y su incidencia en el futuro.

7) La variable social constata otras preocupaciones ya manifestadas relacionadas con el futuro del archivo; sus factores clave se centran en la finalidad del servicio, en la conservación y gestión del objeto y ante todo, en su perspectiva de servicio a la sociedad, colaborando en el logro y mantenimiento de una adecuada "gobernanza" en las administraciones. 
8) De los 70 factores identificados destacan: la necesidad de una formación especializada; la necesidad de gestionar y conservar los objetos (los documentos, electrónicos y convencionales) para su disposición en la organización y como memoria patrimonial; la necesidad de materializar la utilidad del archivo como servicio a la sociedad y garante de un buen gobierno. La necesidad de cambiar la imagen y reconocimiento e la profesión y del archivo.

9) Desde esta investigación se plantea como necesaria su continuidad ampliando su alcance y, en consecuencia, el número y la naturaleza de los expertos participantes. De igual modo se desvela la continuidad del proceso prospectivo, investigando aquellas dos fases que se enunciaron al principio: la definición de tendencias y el diseño de escenarios de futuro.

\section{Bibliografía citada}

Bearman, David. Electronic evidence: strategies for managing records in contemporary organizations. Pittsburgh, PA: Archives and Museum Informatics, 1994.

http://www.archimuse.com/publishing/electronic_eviden ce/ElectronicEvidence.Intro.pdf

Borglund, Erik. "Operational use of electronic records in police work". Information research, 2005, v. 10, n. 4 http://informationr.net/ir/10-4/paper236.html

Brothman, Brien. "Órdenes de valores: cuestionando los términos teóricos de la práctica archivística". Combates por la memoria: archivística de la posmodernidad, Tabula, 2007, n. 10 , pp. 27-58.

Cook, Terry. "Archival science and postmodernism: new formulations for old concepts". Archival science, 2001, v. 1, n. 1, pp 3-24.

Cook, Terry. "Imposturas intelectuales o renacimiento profesional: posmodernismo y práctica archivística". Combates por la memoria: archivística de la posmodernidad, Tabula, 2007, n. 10 , pp. 59-82.

Duranti, Luciana. "The impact of technological change on archival theory". En: The Intl Council on Archives Conf, Sept 16, 2000, Sevilla, Spain.

http://www.interpares.org/documents/ld_sevilla_2000.pdf

Gilliland-Swetland, Anne J. Enduring paradigm, new opportunities: the value of the archival perspective in the digital environment. Washington, DC: Council on Library and Information Resources, 2000.

http://www.clir.org/pubs/reports/pub89/pub89.pdf

Godet, Michel. Manuel de prospective estratégique. Tome 1: Une discipline intellectuel. Paris: Dunod, 2004. ISBN: 9782100512812

Godet, Michel. La caja de herramientas de la prospectiva estratégica. Prospektiker, 2000, cuaderno n. 5.

Godet, Michel; Roubelat, Fabrice. "Creating the future: the use and misuse of scenarios". Long range planning, 1996, abril, v. 29, n. 2, pp. 164-172.

Greene, Mark A. "La fuerza del significado: la misión de los archivos en la era posmoderna". Combates por la memoria: archivística de la posmodernidad, Tabula, 2007, n. 10, pp. 195-212.

Harris, Verne. "Un haz de oscuridad: Derrida en el archivo" Combates por la memoria: archivística de la posmodernidad, Tabula, 2007, n. 10, pp. 123-136.

Hillmann, Karl-Heinz. Diccionario enciclopédico de sociología. Barcelona: Herder Editorial, 2005.

Jimerson, Randall C. "Comprender el poder de los archivos". Combates por la memoria: archivística de la posmodernidad, Tabula, 2007, n. 10, pp. 237-253.

Ketelaar, Erik. "Narrativas tácitas: los significados de los archivos". Combates por la memoria: archivística de la posmodernidad, Tabula, 2007, n. 10, pp. 137-148.

Martín-Pozuelo María-Paz. "Los entornos y documentos electrónicos. ¿Efecto Edison para la formación archivística?”. El reto electrónico: nuevas necesidades, nuevos profesionales. Actas de las V Jornadas de archivos electrónicos. Priego de Córdoba: Ayuntamiento, 2002, pp. 51-64.

Martín-Pozuelo María-Paz. "Prospectiva archivística: nuevas cuestiones, enfoques y métodos de investigación científica". Revista española de documentación científica, 2010, v. 33, n. 2, pp. 201-224.

DOI: $10.3989 /$ redc.2010.2.731

http://redc.revistas.csic.es/index.php/redc/article/viewAr ticle/552

Medina-Vásquez, Javier; Ortegón, Edgar. Manual de prospectiva y decisión estratégica: bases teóricas e instrumentos para América Latina y el Caribe. Santiago de Chile: IELS, 2006, Manuales, n. 51, p. 138, 435 pp.

http://www.oei.es/salactsi/manual51.pdf

Nesmith, Tom. "Una visión de los archivos: posmodernismo y cambio del espacio intelectual de los archivos". Combates por la memoria: archivística de la posmodernidad, Tabula, 2007, n. 10, pp. 213-235.

Palop, Fernando; Vicente, José-Miguel. Vigilancia tecnológica e inteligencia competitiva. Su potencial para la empresa española. Fundación Cotec, 1999, Estudio 15.

http://www.delfos.co.cu/boletines/bsa/PDF/potencial_ vtec.pdf

Ribeiro, Fernanda. "Archival science and changes in the paradigm". Archival science, 2001, v. 1, n. 3, pp. 295-310.

Ringland, Gill. Scenario planning: Managing for the future. Chichester: John Wiley \& Sons, 1998.

Schwartz Peter. "La planification stratégique par scénarios", Futuribles, 1993, mai, n. 176.

Serra, Jordi. "Imaginar el mañana”. La Vanguardia. Ciencia y salud.

http://hemeroteca.lavanguardia.es/preview/1997/01/11/ pagina-6/34616269/pdf.html

Van Der Heijden, Kees. Scenarios: The art of strategic conversation. Chichester \& New York: John Wiley \& Sons, 1996.

Waardenburg, Jean J. Classical approaches to the study of religion: aims, methods, and theories of research. Walter de Gruyter, 1999. 


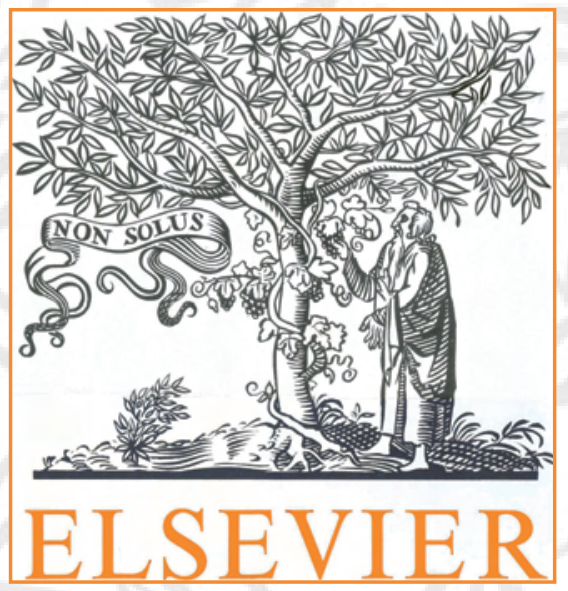

\section{FECYT \\ GESTIONA \\ LA ADQUISICIÓN DE SCIVERSE SCOPUS}

Anunciado el 25 de enero de 2011, la Fundación española para la Ciencia y la Tecnología (Fecyt), dependiente del Ministerio de Ciencia e Innovación (Micinn), compró el acceso online a la base de datos Scopus hasta 2013 para todos los institutos del CSIC, las universidades y centros de investigación públicos españoles.

La posición de Fecyt como interlocutor privilegiado con todos los agentes del Sistema Español de Ciencia y Tecnología, así como la colaboración del Consejo Superior de Investigaciones Científicas (CSIC), la Conferencia de Rectores de Universidades Españolas (CRUE), los directores de los consorcios bibliotecarios universitarios, grupos de compra y bibliotecas virtuales de sanidad, ha hecho posible el acuerdo, que implica un $50 \%$ de descuento sobre las tarifas oficiales de Sciverse Scopus (un ahorro de más de 4 millones de euros).

Como se sabe, SciVerse tiene dos componentes: Science Direct, que son artículos de revistas en texto completo, y Scopus, que es una base de datos bibliográfica con abstracts y bibliografía citada que a fecha de hoy contiene 42,5 millones de registros. Aquí estamos hablando de Scopus.

Preguntamos a Javier Arenas, de Elsevier, si ha sido fácil la negociación:

- Es importante reflejar que este acuerdo se ha conseguido por la buena voluntad de los anteriores suscriptores de Scopus, más de 28 instituciones incluyendo al CSIC. Elsevier ya inició contactos hace más de 4 años con el Ministerio, y en Amsterdam han entendido que en la situación económica actual y para lograr una mayor homogeneidad y estabilidad en los contratos, la oportunidad brindada era la adecuada. Se ha bajado el precio de una manera razonable para ambas partes, que sólo se entiende en este contexto, nacional y amparados por la figura del Ministerio de Ciencia e Innovación, la CRUE y los consorcios de bibliotecas, universitarias y de las consejerías. La actuación de la Fecyt, que ha sabido ponernos juntos a todos y de acuerdo, ha sido providencial.

\section{¿Qué planes tiene ahora Elsevier?}

- Elsevier sigue invirtiendo en Scopus y hace protagonistas a sus usuarios para

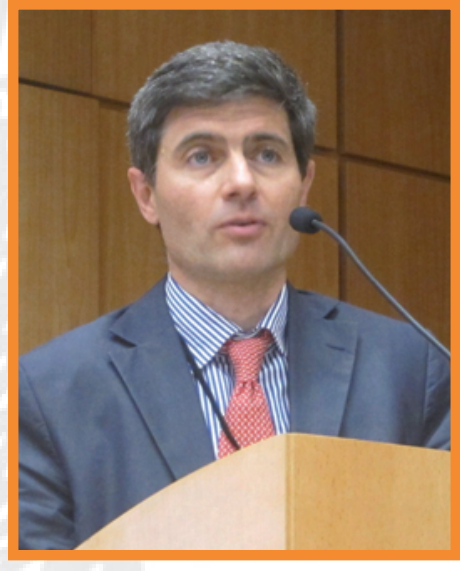

Javier Arenas-Ybarra gerente para Instituciones, Elsevier BV mejorarlo juntos y que se pueda sacar el máximo partido de la herramienta. Ahora estamos trabajando para la promoción y formación de Scopus y conseguir que el mayor número posible de instituciones se adhieran al proyecto, que se ha diseñado de tal forma que está abierto a toda la comunidad académica y a las instituciones gubernamentales de investigación (nacionales o regionales), no importando su tamaño.

\section{¿Llegará SJR a sustituir el FI de ISI?}

- No creo que de momento se produzca un cambio radical en los patrones actuales de medir la calidad de las publicaciones científicas. Lo que sí que es cierto es que Sciverse Scopus compite con muchas más revistas (18.000, publicadas por más de 5.000 editores internacionales) y además incluye 23 millones de patentes, 3,7 millones de ponencias y 435 millones de sitios web científicos. Nosotros creemos que las dos métricas de impacto de la investigación: Scimago journal rank (SCR) y Source normalized impact per paper (SNIP) -de uso gratuito- son más adecuadas, amen que Scopus cubre mejor las revistas europeas y las ciencias sociales. Sin duda estamos avanzando hacia esa posibilidad y la ciencia española ha dado un gran paso en esa dirección. 